\title{
Single-Step Data-Domain Least-Squares Reverse-Time Migration Using Gabor Deconvolution for Subsalt Imaging
}

\author{
Qiancheng Liu ${ }^{(}$, Member, IEEE, Yongming Lu, Hui Sun, and Hao Zhang
}

\begin{abstract}
Least-squares reverse-time migration (LSRTM) distinctly improves seismic imaging quality, but at an expensive computation overhead involving tens of iterations. We herein take a computationally cheaper single-step LSRTM solution, which intrinsically performs deblurring through a data-domain Wiener deconvolution. However, the Wiener filter mainly solves the signal estimation problems for stationary signals. Subsalt imaging often suffers from strong salt-related reflections and artifacts. The former and the latter give rise to strong amplitude variance and changed source wavelets in the demigrated data, increasing its nonstationarity and hindering the data-deblurring operation in the single-step LSRTM. To alleviate the nonstationarity during the data-domain deblurring, we consider a Gabor deconvolution method. Testing on the Sigsbee data sets shows that the Gabor deconvolution method is effective, producing subsalt images of more balanced events and fewer artifacts than the raw RTM image. The Gabor deconvolution-related result also outperforms the standard single-step LSRTM result with more robust behavior and better subsalt imaging quality.
\end{abstract}

Index Terms-Deconvolution, Gabor transform, least-squares imaging, subsalt.

\section{INTRODUCTION}

$\mathbf{R}$ EVERSE-TIME migration (RTM) acts as the stateof-the-art imaging method for complex subsurface structures [1], [2]. RTM has also got applications to ground-penetrating radar imaging [3] and electromagnetic imaging [4]. RTM, however, remains an adjoint operator rather than the inverse operator of the forward modeling. The resulting RTM images may suffer from band-limited source effect and uneven amplitudes. Least-squares RTM (LSRTM) approximates the inverse operator [5], promising better imaging quality and resolution.

Manuscript received November 27, 2018; revised February 2, 2019 and March 24, 2019; accepted April 13, 2019. This work was supported by the King Abdullah University of Science and Technology (KAUST).(Corresponding author: Qiancheng Liu.)

Q. Liu is with the Department of Physical Sciences and Engineering, King Abdullah University of Science and Technology, Thuwal 23955-6900, Saudi Arabia (e-mail: qiancheng.liu@kaust.edu.sa).

Y. Lu is with the Department of Earth and Space Sciences, Southern University of Science and Technology, Shenzhen 518055, China (e-mail: luym@sustc.edu.cn).

H. Sun is with the Institute of Geology and Geophysics, Chinese Academy of Sciences, Beijing 100029, China (e-mail:sh39o@mail.ustc.edu.cn).

H. Zhang is with the Institute of Geomechanics, Chinese Academy of Geological Sciences, Beijing 100081, China (e-mail: zhanghao@geomech.ac.cn).

Color versions of one or more of the figures in this letter are available online at http://ieeexplore.ieee.org.

Digital Object Identifier 10.1109/LGRS.2019.2916847
LSRTM has gained plenty of attention since being proposed. Within an inversion workflow, LSRTM attempts to refine the RTM image toward the reflectivity imaging, iteratively [5], [6] or single-step [7], [8]. Compared with the iterative LSRTM, the single-step approaches, which strike a balance between imaging quality and computational cost, provide an appealing solution through either image-domain or data-domain deblurring. Here, we focus on the application of single-step datadomain LSRTM [8] to subsalt imaging.

With the presence of large velocity contrasts and steep dips, salt body poses a challenge in front of geophysicists. For this reason, subsalt imaging usually suffers from strong salt-related reflections and artifacts, for example, the salt halo, which is a type of noise along salt franks. These kinds of artifacts degrade the image around the salt body. After the demigration operation, the image-domain artifacts turn into data-domain artifacts. Together with the strong amplitude variance caused by the velocity contrasts around the salt body, these artifacts, such as salt halo, distort the source wavelets on the reflectors, leading to nonstationarity issues within the conventional deblurring process. To alleviate the nonstationarity [9]-[11], we propose a Gabor deconvolution approach. This solution is a direct extension of the Wiener deconvolution [8], which mainly solves for stationary signals, simply with an additional Gabor transform before the deconvolution operation at a negligible overhead.

This letter is organized as follows. First, we briefly review the single-step data-domain LSRTM. Then, we propose the Gabor deconvolution in the data-domain deblurring. Finally, we validate our methods using the Sigsbee data set.

\section{Methods}

\section{A. Review of Single-Step Data-Domain LSRTM}

LSRTM mainly comprises two operators: 1) demigration operator by Born modeling and 2) migration operator by RTM. Both the operators are based upon the linearized two-way wave equation, thus have no dip limitation. For simplicity, we represent the operators below in matrix-vector notations.

The demigration operator is based on Born modeling, a firstorder approximation to the full wave equation, as follows:

$$
\mathbf{L m}=\mathbf{d}
$$

where the forward operator $\mathbf{L}$ is related to a migration velocity, a specific observation system, and a given source wavelet, $\mathbf{m}$ is the reflectivity model, and $\mathbf{d}$ the observed data. 
The migration operator is based on the adjoint method, from which we can obtain the RTM image as follows:

$$
\mathbf{m}_{\text {mig }}=\mathbf{L}^{T} \mathbf{d}=\mathbf{L}^{T} \mathbf{L} \mathbf{m}
$$

in which $\mathbf{L}^{T}$ denotes the adjoint operator (i.e., RTM operator) regarding the forward operator $\mathbf{L}$. From (2), we notice that the term $\mathbf{L}^{T} \mathbf{L}$ acts as the Hessian acting on the reflectivity model $\mathbf{m}$, resulting in a blurred image $\mathbf{m}_{\text {mig }}$. The goal of LSRTM is to solve for the reflectivity model $\mathbf{m}$ in a least-squares sense. The Hessian, however, is prohibitive to compute or store for practical problems. Conventional LSRTM attempts to approximate the inverted reflectivity model by using gradient optimization methods. Even though involving tens of iterations, iterative LSRTM remains costly in computation.

Liu and Peter [8] observed that there might exist some data-domain solution through the following approximation:

$$
\mathbf{m}=\left(\mathbf{L}^{T} \mathbf{L}\right)^{-1} \mathbf{L}^{\mathbf{T}} \mathbf{d} \approx \mathbf{L}^{T}\left(\mathbf{L} \mathbf{L}^{T}\right)^{-1} \mathbf{d}
$$

which is an approximation to $\left(\mathbf{L}^{T} \mathbf{L}+\lambda\right)^{-1} \mathbf{L}^{\mathbf{T}}=$ $\mathbf{L}^{T}\left(\mathbf{L} \mathbf{L}^{T}+\lambda\right)^{-1}$, with $\lambda$ being a regularization term. When the data-misfit contribution is mainly emphasized, the relationship in (3) holds, which indicates a straightforward manner to approximate the reflectivity image once the observed data $\mathbf{d}$ has been preconditioned by $\mathbf{F}=\left(\mathbf{L} \mathbf{L}^{T}\right)^{-1}$. The term $\mathbf{F}$ can be estimated in an $L 2$-norm misfit function

$$
e(\mathbf{F})=\left\|\mathbf{F} \mathbf{d}_{1}-\mathbf{d}\right\|_{2}^{2} .
$$

Under a trace-by-trace approximation [8], one can determine F by

$$
\mathbf{F}(\omega, \mathbf{r})=\frac{\mathbf{d}(\omega, \mathbf{r}) \mathbf{d}_{1}^{*}(\omega, \mathbf{r})}{\mathbf{d}_{1}(\omega, \mathbf{r}) \mathbf{d}_{1}^{*}(\omega, \mathbf{r})}=\frac{\mathbf{d}(\omega, \mathbf{r}) \mathbf{d}_{1}^{*}(\omega, \mathbf{r})}{\left|\mathbf{d}_{1}(\omega, \mathbf{r})\right|^{2}+\varepsilon}
$$

where $\varepsilon$ is a stabilizer, $\omega$ is the angular frequency, and $\mathbf{r}$ is the receiver position. Equation (5) is a Wiener deconvolution operation. For the sake of stabilization, we adopt a stabilized division algorithm in [12] for (5). After putting $\mathbf{F}$ back to (3), one can approximate the deblurred image, i.e., the inverted reflectivity result. In summary, the single-step data-domain LSRTM consists of four steps: 1) RTM by (2); 2) demigration by (1) from the RTM image; 3 ) estimation of the deblurring preconditioner by (5); and 4) remigration of the preconditioned observed data by (3). The total computational cost of the single-step data-domain LSRTM is around two RTMs. Note that in practical applications, one does not need to output the preconditioner $\mathbf{F}$ but directly imposes it onto the observed data $\mathbf{d}(\omega, \mathbf{r})$. The overhead arising from (5) is negligible compared with that from seismic modeling.

\section{B. Gabor Deconvolution in Data-Domain Deblurring}

The preconditioner in (5) works trace by trace, which suffices for general applications. When it comes to subsalt imaging, however, merely the trace-by-trace solution may not be enough. The presence of strong velocity contrasts of complex geometries around the salt body poses a variety of challenges for subsalt imaging. On the one hand, the strongvelocity contrasts act as strong reflectors, leading to strong amplitude unbalance and poor subsalt illumination; on the other hand, there may exist some "salt halo" artifacts along

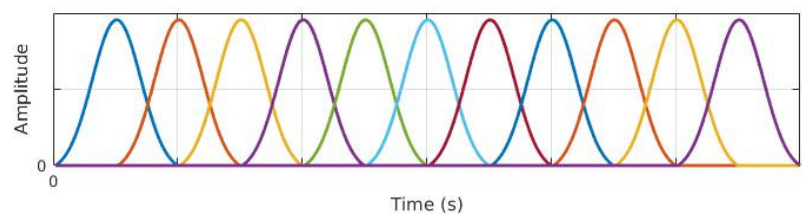

Fig. 1. Gaussian windows used in Gabor deconvolution. The window increment is at half of the window length.

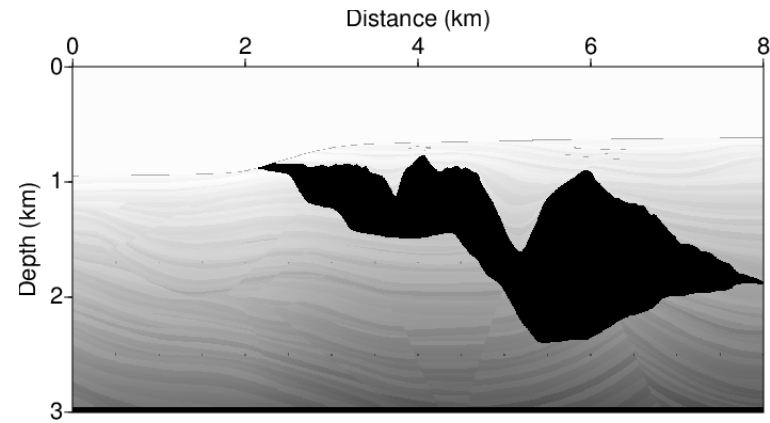

(a)

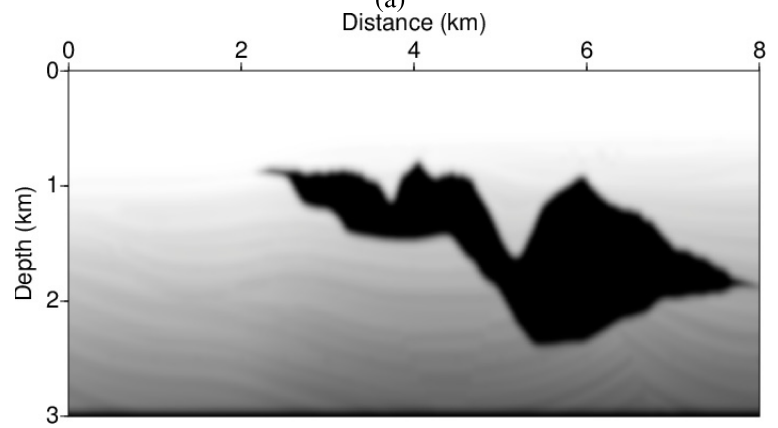

(b)

Fig. 2. (a) True Sigsbee model used for the generation of observed data. (b) Smoothed Sigsbee model used for migration and demigration. (b) is obtained by blurring (a) with a 2-D Gaussian smoother to remove the high-wavenumber details.

the salt boundaries, which turn into unwelcomed artifacts in the data domain after demigration. They both increase the nonstationarity level of the seismograms, exhibiting challenges in front of the trace-based Wiener deconvolution, which is more suitable for stationary signals.

To mitigate the nonstationarity by solving (5) and for a better deblurring purpose, we seek the help of Gabor deconvolution, (Fig. 1), deconvolution done via Gabor transform, which is a particular case of the short-time Fourier transform. The Gabor transform decomposes a seismogram into a time-frequency plane. The signals to be transformed are time-windowed with Gaussian functions, and the resulting signals are then Fourier transformed for the time-frequency analysis. For one Salt seismogram trace with distinct variance in reflectivity, it may be globally nonstationary but locally stationary. Gabor deconvolution, thus, reduces the nonstationarity within the local data-domain deblurring process. For a given signal $s(t)$, its Gabor transform is defined as

$$
G_{s}\left(t_{k}, \omega\right)=\int s(t) g_{k}(t) e^{-i \omega t} d t
$$



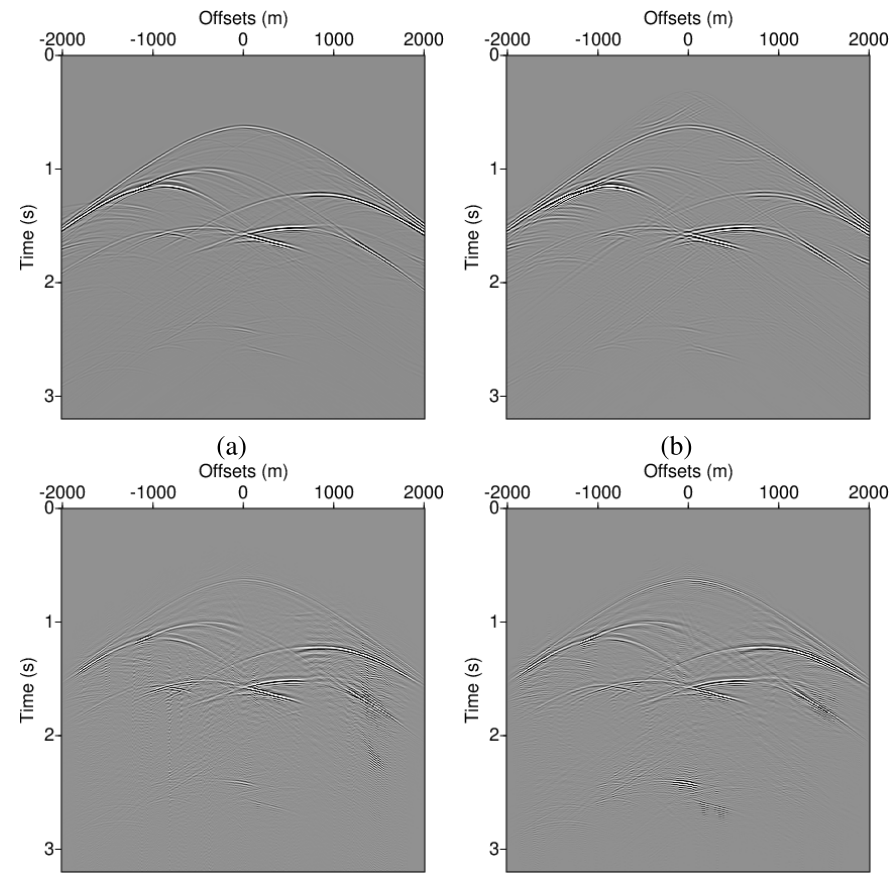

(c)

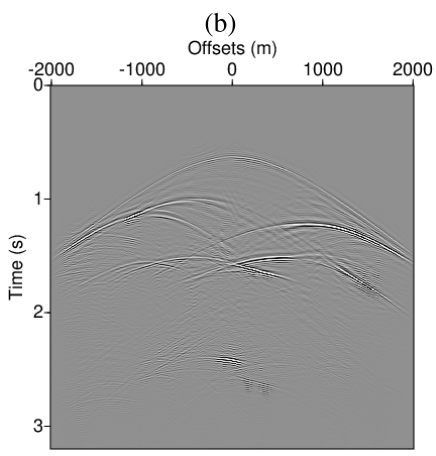

(d)

Fig. 3. Common-shot gathers of source location at $5000 \mathrm{~m}$. (a) Observed data. (b) Demigrated data from RTM image. (c) Data-domain deblurred data by Wiener deconvolution. (d) Data-domain deblurred data by Gabor deconvolution. In (c), we can see that some spurious artificial events extend away from the seismic events. These artifacts are caused by the salt-related nonstationarities, such as the changed source wavelets within the halo artifacts and migration artifacts around the salt body, during Wiener deconvolution. As a nonstationary operation, the Gabor deconvolution in (d) performs the data-domain deblurring without long-lasting artifacts as those in (c). Also, (d) shows that Gabor deconvolution has better time resolution in amplitude balancing, especially for the two deeper events around $2.5 \mathrm{~s}$.

in which $g_{k}(t)$ is a Gaussian window, and $k$ indexes the Gaussian window. Given $G_{s}\left(t_{k}, \omega\right)$, the signal can be reconstructed via

$$
s(t)=\gamma(t) \int G_{s}\left(t_{k}, \omega\right) e^{i \omega t} d \omega
$$

with $\gamma(t) \sum_{k} g_{k}(t)=1$ for an amplitude balancing purpose. Equation (7) shows that a strict Gabor transform may need normalization of the summed weight of the Gaussian windows, especially for the two ends of $s(t)$, from the energy conservation point of view. For seismic data, however, it is reasonable to neglect the normalization operation because the signals at the two ends usually are invalid or even zero. For the sake of numerical stability, the Gabor transform requires an oversampling criterion of $d f * d t<1$. This property is related to the Heisenberg uncertainty principle. Note that the best simultaneous resolution of time and frequency is reached with a Gaussian window function in the Gabor transform.

Recalling (5), in which we perform our deconvolution under the frame of Gabor transform, results in the so-called Gabor deconvolution. That is, we put forward the window-by-window operation within the trace-by-trace operation in preconditioner estimation

$$
\mathbf{F}_{(g)}(\omega, \mathbf{r})=\frac{\mathbf{d}_{(g)}(\omega, \mathbf{r}) \mathbf{d}_{1(g)}^{*}(\omega, \mathbf{r})}{\left|\mathbf{d}_{1(g)}(\omega, \mathbf{r})\right|^{2}+\varepsilon}
$$

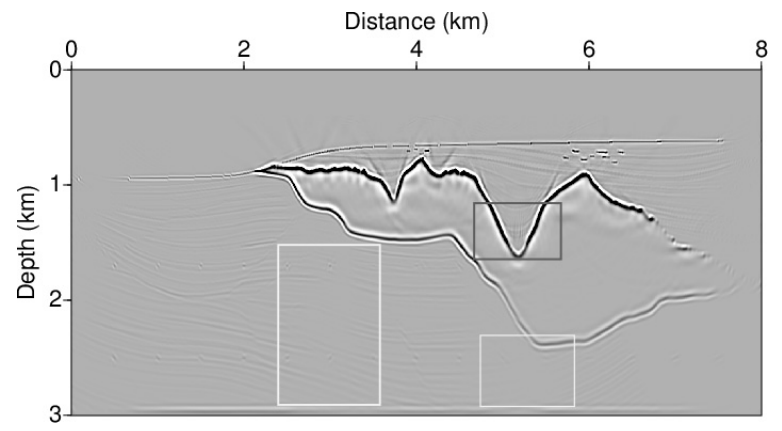

(a)

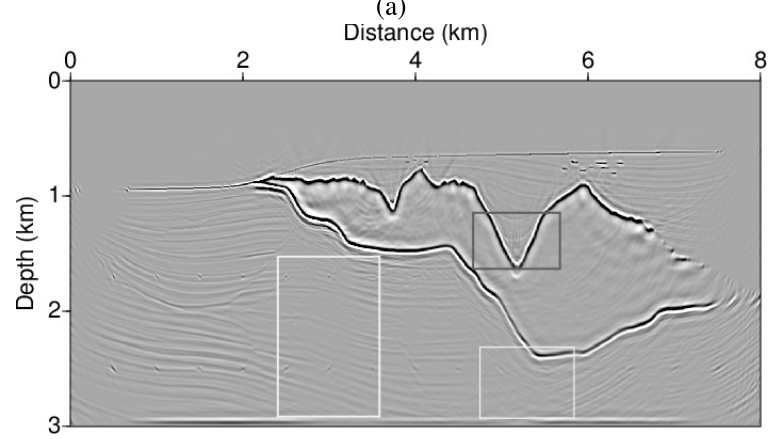

(b)

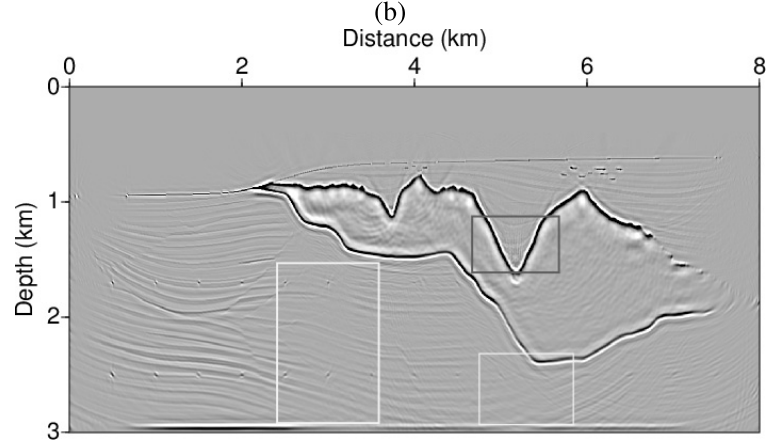

(c)

Fig. 4. (a) Normalized RTM image. The strong salt-related reflections and the halo artifacts around the salt body give rise to strong amplitude variance and altered source wavelets for the reflectors, increasing the nonstationarities in the demigrated data. (b) Conventional single-step LSRTM image by Wiener deconvolution. (c) Improved single-step LSRTM image by Gabor deconvolution. Both (b) and (c) outperform (a). Compared with (b), (c) has a better amplitude illumination for subsalt areas and fewer artifacts around the salt body, as marked by the boxes, thanks to the Gabor deconvolution in Fig. 3(d). Also, we see that the halo artifacts around the salt upper boundaries have been reduced. However, note that there remain some "stripe" artifacts within the gray boxes, which we will look into in the future study.

in which the subscript $(g)$ denotes (8) operates within a Gaussian window. Note that we do not need to obtain $\mathbf{F}_{(g)}$ as well but directly impose it upon $\mathbf{d}_{(g)}$, yielding $\widetilde{\mathbf{d}}_{(g)}$. Stacking $\widetilde{\mathbf{d}}_{(g)}$ over all the windows results in $\widetilde{\mathbf{d}}$. After inverse Fourier transforming $\widetilde{\mathbf{d}}$, one can remigrate the time-domain preconditioned seismograms for a deblurred image. This way, we expect to take advantage of Gabor deconvolution to mitigate the nonstationarity in the data-domain deblurring of single-step LSRTM.

\section{NUMERICAL EXAMPLE}

We test our methods upon the 2-D Sigsbee synthetic data. The observed data are generated from the true velocity model in Fig. 2(a), with direct waves muted. There are 125 shots 


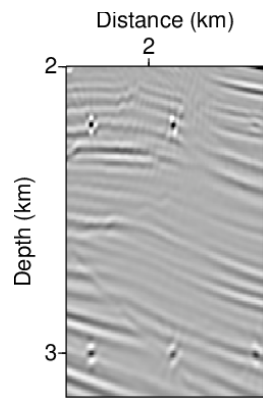

(a)

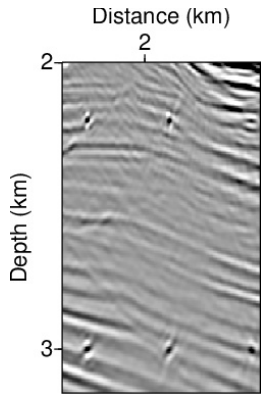

(b)

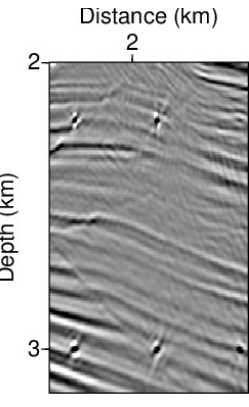

(c)
Fig. 5. (a)-(c) Representative zoomed-in views of the three left-side white boxes (the right-side ones not so informative) from Fig. 4. (b) has more continuous reflectors than (a), but with unexpected artifacts caused by Wiener filtering for nonstationary around-salt signals. Compared with (a) and (b), (c) has the best imaging quality, thanks to the Gabor filtering, which make the data-domain deblurring locally stationary.

evenly distributed from 1000 to $7200 \mathrm{~m}$. The source signature is a Ricker wavelet with a central frequency of $20 \mathrm{~Hz}$. The fixed-spreading observation system consists of 801 receivers spacing at a $10-\mathrm{m}$ interval. The simulation duration is $5 \mathrm{~s}$ in total. Fig. 2(b) shows the migration velocity obtained from a 2-D Gaussian smoother, with high-wavenumber features removed, but we notice that the strong velocity gradient around the salt body remains preserved.

From the Common-Shot-Gather (CSG) in Fig. 3(a), we obtain the RTM image in Fig. 4(a), which has been normalized by source illumination. The salt body gets imaged well, but with salt-related artifacts. The subsalt imaging areas are weakly resolved due to unbalanced source illumination. To perform the single-step data-domain LSRTM, we hereafter demigrate from Fig. 4(a) for the data in Fig. 3(b). Compared with Fig. 3(a), the seismograms shown in Fig. 3(b) suffer from unbalanced amplitudes and degraded time resolution. These effects can be taken as the Hessian effects mapped from the image domain to the data domain. We first try the conventional single-step data-domain LSRTM. The direct Wiener deconvolution yields the preconditioned data in Fig. 3(c), which contains artifacts around the salt reflections. Fig. 4(b) shows its corresponding single-step LSRTM image. It achieves the deblurring effect, but together with the presence of additional migration artifacts around the salt body. We suppose more actions can be done for further improvements in Fig. 4(b).

We, subsequently, attempt to improve the single-step LSRTM image by Gabor deconvolution. The key idea of this approach is to perform data-domain deblurring within a Gabor transform. We choose a Gaussian window length of $1 \mathrm{~s}$ for Gabor transform, with an increment of $0.5 \mathrm{~s}$ along time-windowed signals. The Gaussian windows start from the first effective signal per trace. The determination of an optimal window length (and increment) is elusive, so here we leave it as an open question. In Fig. 3(d), we can see that the Gabor deconvolution also achieves a strong deconvolution effect but with no presence of filtering artifacts around the salt reflections. The remigrated image from the new preconditioned seismogram is shown in Fig. 4(c), which has achieved the deblurring effect as well, without weakly introduced migration artifacts around the salt body. Compared with the conventional RTM, the two single-step LSRTM methods produce the subsalt image of higher resolution, more balanced amplitudes, and more coherent events, as marked by white boxes. Also, we pick out the three representative zoomed-in views from the left-side white boxes for detailed investigations, as shown in Fig. 5. If we compare the two single-step ones, the Gabor deconvolution-related method is more powerful and robust.

\section{CONCLUSION}

Data-domain single-step LSRTM is effective in deblurring RTM images through preconditioning. When it comes to subsalt imaging, the trace-by-trace operation may fail due to the increased nonstationarity caused by unbalanced subsalt illumination and the salt-related artifacts. Note that the nonstationarity here is related to the distinct variance in reflectivity rather than source wavelet. To mitigate this nonstationary, we propose to perform the data-domain deblurring operation with the Gabor deconvolution for each trace. Our method is a direct extension of the Wiener filtering in the Fourier domain. This way, we can achieve the deblurring effect but without artifacts caused by the conventional operation. Our method shows promising results in subsalt imaging. It improves the subsalt imaging resolution as well as compensates for the unbalanced illumination, without the introduction of additional migration artifacts around the salt body. Even at a cheap computational cost of single step rather than tens of iterations, our method provides a practical solution by using Gabor deconvolution for subsalt imaging.

\section{ACKNOWLEDGMENT}

The authors would like to thank the Information Technology Division and Extreme Computing Research Center (ECRC) at the King Abdullah University of Science and Technology (KAUST) for providing resources for computer time.

\section{REFERENCES}

[1] E. Baysal, D. D. Kosloff, and J. W. Sherwood, "Reverse time migration," Geophysics, vol. 48, no. 11, pp. 1514-1524, 1983.

[2] N. D. Whitmore, "Iterative depth migration by backward time propagation," in SEG Technical Program Expanded Abstracts. Society of Exploration Geophysicists, 1983, pp. 382-385.

[3] F. Foroozan and A. Asif, "Time-reversal ground-penetrating radar: Range estimation with Cramér-Rao lower bounds," IEEE Trans. Geosci. Remote Sens., vol. 48, no. 10, pp. 3698-3708, Oct. 2010.

[4] M. E. Yavuz and F. L. Teixeira, "Frequency dispersion compensation in time reversal techniques for UWB electromagnetic waves," IEEE Geosci. Remote Sens. Lett., vol. 2, no. 2, pp. 233-237, Apr. 2005.

[5] T. Nemeth, C. Wu, and G. T. Schuster, "Least-squares migration of incomplete reflection data," Geophysics, vol. 64, no. 1, pp. 208-221, 1999.

[6] X. Liu, Y. Liu, H. Lu, H. Hu, and M. Khan, "Prestack correlative least-squares reverse time migration," Geophysics, vol. 82, no. 2, pp. S159-S172, 2017.

[7] A. Guitton, "Amplitude and kinematic corrections of migrated images for nonunitary imaging operators," Geophysics, vol. 69, no. 4, pp. 1017-1024, 2004.

[8] Q. Liu and D. Peter, "One-step data-domain least-squares reverse time migration," Geophysics, vol. 83, no. 4, pp. 1-46, 2018.

[9] J. B. Tary, R. H. Herrera, J. Han, and M. van der Baan, "Spectral estimation-What is new? What is next?" Rev. Geophys., vol. 52, no. 4, pp. 723-749, 2014.

[10] S. Yuan, S. Wang, M. Ma, Y. Ji, and L. Deng, "Sparse Bayesian learningbased time-variant deconvolution," IEEE Trans. Geosci. Remote Sens., vol. 55, no. 11, pp. 6182-6194, Nov. 2017.

[11] S. Yuan, Y. Ji, P. Shi, J. Zeng, J. Gao, and S. Wang, "Sparse Bayesian learning-based seismic high-resolution time-frequency analysis," IEEE Geosci. Remote Sens. Lett., vol. 16, no. 4, pp. 623-627, Apr. 2019.

[12] Q. Liu, J. Zhang, and H. Zhang, "Eliminating the redundant source effects from the cross-correlation reverse-time migration using a modified stabilized division," Comput. Geosci., vol. 92, pp. 49-57, Jul. 2016. 